\title{
Simple or complex? A case study of physical and mental health co-morbidity
}

\author{
Sophie D. Bennett ${ }^{1}$, Isobel Heyman ${ }^{1,2}$, Sophia Varadkar ${ }^{1,2}$, Anna E. Coughtrey ${ }^{1,2}$ and \\ Roz Shafran ${ }^{1}$ \\ ${ }^{1}$ University College London, Institute of Child Health, 30 Guilford Street, London WC1N 1EH \\ ${ }^{2}$ Great Ormond Street Hospital for Children NHS Foundation Trust, Great Ormond Street, London \\ WC1N $3 J H$
}

Received 28 July 2016; Accepted 30 June 2017

\begin{abstract}
People with epilepsy are significantly more likely to have a mental health disorder than those without a chronic illness. The reasons for this are multiple but may include the mental health difficulties being perceived as complex due to the presence of a chronic illness. In part due to the apparent complexity of the co-occurring physical and mental illness, many are not offered evidence-based treatment (EBT) for the mental health disorder. There is little guidance to inform clinicians about the interventions to use to treat mental health disorders in people with epilepsy. The present paper reports a case of treatment for depression using a standard EBT in a young person with epilepsy. The patient also had clinically significant symptoms of anxiety and an eating disorder and would be considered 'complex' according to standard criteria. The intervention, however, was relatively simple and was delivered as guided self-help via 10 weekly telephone calls of approximately 30 minutes duration, and two follow-up calls at one month and three months post-intervention. Self-report and parent-report questionnaire measures were completed before and after the intervention, and at both follow-up time points. A blind-rated online diagnostic interview measure was also completed before and after the intervention. The young person and her family also completed a qualitative interview of their experiences of the intervention. This simple intervention was effective in working towards the client's goals, although pre-post measurement on standard measures was variable. This interesting case raises questions about whether patients with mental and physical comorbidities are complex, or just perceived as complex.
\end{abstract}

Key words: CBT, self-help, depression, children and adolescents

\section{Introduction}

Psychological interventions for mental health disorders have consistently demonstrated efficacy in hundreds of randomized controlled trials; a recent overview of meta-analyses found a mean effect size of 0.58 (Huhn et al. 2014). Cognitive behavioural therapy (CBT),

Author for correspondence: Sophie Bennett, University College London, Institute of Child Health, 30 Guilford Street, London WC1N 1EH. (E-mail: sophie.bennett.10@ucl.ac.uk) 
in particular, has been shown to be efficacious for the treatment of anxiety disorders and eating disorders (Hofmann et al. 2012). As a result, CBT is cited by NICE guidelines as the first-line treatment of choice for both adults and children with anxiety disorders and depression (e.g. NICE, 2005, 2009, 2011). Yet many people with mental health disorders do not receive evidence-based intervention (Prince et al. 2007). The reasons for this are multiple, but may include clinicians' beliefs that patients in clinical practice have more severe and complex problems than participants in research, due to strict exclusion criteria in clinical trials, as well as the belief that research trials are better resourced than clinical settings (Lilienfeld et al. 2013; Shafran et al. 2009; Stirman et al. 2004).

People with chronic health conditions typically fall into the category of those who are often excluded from clinical trials (cf. James et al. 2005) as they are considered to be complex. For example, the All Party Parliamentary Group for Complex Needs and Dual Diagnoses defines a person with complex needs as 'someone with two or more needs affecting their physical, mental, social or financial wellbeing' (Complex Needs and Dual Diagnosis APPG. 2014, June. Factsheet 1: Complex needs and dual diagnosis. Retrieved from http://www.turning-point.co.uk/media/636823/appg_factsheet_1_-_june_2014.pdf). They state that a person with both mental and physical health conditions would be considered to have complex needs.

People with chronic illness are at higher risk of developing mental health difficulties than those without. Mental health difficulties are associated with increased morbidity and mortality in those with physical illness (e.g. Katon, 2003; Katon et al. 2004; Kovacs et al. 1995; Lespérance et al. 2002; Whooley et al. 2008). Those with chronic neurological conditions, such as epilepsy, are at particularly high risk, with rates up to $54.5 \%$ in community samples (see Tellez-Zenteno et al. 2007, for review).

There are likely to be multiple pathways to the development of mental health difficulties in people with epilepsy and hence reasons for the increased prevalence. For example, in depression, a diathesis-stress model has been described, whereby 'chronic stress exposure owing to the burden of epilepsy and learned helplessness due to the threat of recurrent seizures as unpredictable aversive events represent psychological risk factors' for depression (Hoppe and Elger, 2011). Some anti-epileptic drugs (AEDs) may have mood stabilising properties, whereas others are associated with increased risk of depression (Ettinger, 2006), particularly if the patient has a history of psychiatric illness (Nadkarni and Devinsky, 2005). Shared underlying pathology may also play a role (Heyman et al 2015), particularly as epilepsy has been associated with an increased likelihood of developing a psychiatric disorder even prior to epilepsy diagnosis (e.g. Hesdorffer et al. 2012). Not only may epilepsy be a risk factor for developing depression, but patients with depression also have a three to seven times greater risk of developing epilepsy, suggesting a bidirectional relationship between the two (Kanner, 2011). Additionally, there is an increased prevalence of neurodevelopmental disorders, such as autism spectrum disorders (Clarke et al. 2005), and attention deficit hyperactivity disorder (Dunn and Austin, 2003), intellectual disabilities (Sillanpää, 2004) and specific learning difficulties (Fastenau et al. 2008) in people with epilepsy, which themselves may be risk factors for the development of mental health disorders (Heyman, 2015).

This 'complex interplay between psychological, physical and social factors', coupled with low detection rates, has led to a significant proportion of people with chronic illness not having their mental health needs met (Prince et al. 2007), perhaps in part because they are considered 'difficult to ascertain, diagnose, or treat' (Complex Needs and Dual Diagnosis APPG, 2014). 
This is particularly the case for children and young people with chronic illnesses, including those with neurological conditions. For example, Ott and colleagues (2003) found that of 114 children with epilepsy, $61 \%$ had psychiatric diagnoses, but of these, only $33 \%$ had received treatment, despite regularly attending clinics for their epilepsy. This situation is not unique to neurological conditions; Katon and colleagues (2006) found that only $35 \%$ of young people with asthma and a co-morbid anxiety and/or depressive disorder (identified through screening interviews) were recognised by the medical system as having a mental health disorder. In those young people diagnosed with a co-morbid major depression, only $17 \%$ received four or more psychotherapy sessions. Most recently, the UK Children's Commissioner's Lightning review found that $60 \%$ of mental health conditions in the context of physical health problems go undetected and untreated, whilst the outcome of Child and Adolescent Mental Health Service (CAMHS) referrals was unknown for almost $80 \%$ of 4098 children and young people with emotional difficulties related to their physical conditions (Children's Commissioner, 2016). Whilst it is not known whether the primary reason for a lack of treatment is perceived complexity, research on interventions tailored specifically towards specific chronic illnesses (e.g. McGrady and Hood, 2013; Reigada et al. 2013) suggests that some consider that current evidence-based treatments (EBTs) are either not sufficient, or are not optimal for people with these chronic illnesses, despite the lack of evidence for this assertion.

But why might people assume such patients are complex, and therefore may not respond as well to treatment, simply because they have a chronic illness? As stated by Lau (2006) in consideration of cultural adaptations of EBTs, 'although there is as yet limited evidence supporting the effectiveness of EBTs with minorities, evidence that they are less effective with minorities is likewise limited'. As with cultural adaptations, there has been extremely little research to date investigating treatment for mental health disorders in adults with chronic physical conditions (Hadert, 2013) and even fewer studies in children and young people with chronic physical conditions (Bennett et al. 2015).

Lau and colleagues (2006) further suggest that the first priority is to proceed with deployment to ensure minorities have access to evidence-based care and evaluate parity within inclusive effectiveness trials'. We agree with this sentiment and consider that before embarking on modification of an existing treatment, or even developing a new one, surely it should be determined whether 'simple' evidence-based CBT interventions are effective in this population. Adapting treatments may compromise their fidelity and therefore effectiveness, and could be wasteful of resources (Lau et al. 2006). Given the proven efficacy of 'simple' CBT interventions in the treatment of mental health disorders in a wide range of populations and for a wide range of difficulties, 'there is no logical reason why treatments known to be effective already ... should not also be effective in the presence of chronic illness' in children and young people (Cottrell, 2015). It is therefore most parsimonious to start with these.

Yet, even if we can demonstrate efficacy of these interventions in a complex population, how do we address the second reason for the gap between research findings and clinical practice, namely, the capacity of clinical services? The prevalence of epilepsy alone is $0.97 \%$, equivalent to 600,000 people in the UK. Assuming a prevalence of depression of 30-50\% in this group (Kanner, 2003), of which large numbers do not have access to intervention, this is a significant unmet need. UK clinical practices are already stretched beyond capacity (Lousada et al. 2015). We need to provide simple, cost-effective interventions that can be widely disseminated. 
One way of meeting a large unmet need for psychological therapy is through the use of self-help or guided self-help interventions (the latter involving varying degrees of input from a therapist). This both reduces the impact of stigma (as it is possible to access it without others knowing), and reduces the time burden associated with visiting a clinic. It is cost effective and is as efficacious as face-to-face therapy for the treatment of anxiety and depression (Cuijpers et al. 2010). Guided self-help is now a recommended first-line treatment for mild to moderate anxiety and depression in adults (e.g. NICE, 2009a; NICE, 2011), as well as for adults with a chronic physical health problem (NICE, 2009b). NICE states that the evidence for this guideline was based on evidence from the Depression guidance, and that there is little evidence to conclude its efficacy in people with a chronic physical illness. It therefore calls for research investigating the efficacy of low-intensity interventions for depression in patients with a chronic physical health problem.

This paper reports on a case of a young person with depression and anxiety in the context of severe epilepsy. A short, simple telephone guided self-help CBT intervention was used. The case was evaluated through both quantitative measures and a qualitative interview.

\section{Methods}

Chloe (patient's name and identifying details have been changed to preserve anonymity) was a 17-year-old with epilepsy. This work was completed as part of a research study investigating the feasibility of routine screening and intervention for common mental health difficulties in children and young people with epilepsy.

\section{Intervention}

The intervention used in the research study was based on the Modular Approach to Therapy for Children with Anxiety, Depression, Trauma, or Conduct Problems (MATCH-ADTC) protocol (Chorpita and Weisz, 2009). MATCH-ADTC is a modular-based intervention, with larger modules for the treatment of anxiety, trauma, conduct problems and/or depression. Within these larger modules are 31 smaller stand-alone modules, for example on behavioural activation in the depression module, thought challenging in the anxiety module, or praise in the behaviour module. The modules correspond to the procedures used in the leading evidence-based psychological treatments that have been evaluated in over 322 randomized controlled trials. The three treatments are 'Coping Cat' for anxiety disorders, 'Primary and Secondary Control Enhancement Training' (PASCET) for depression, and Parent Training for disruptive conduct (Barkley et al. 1997; Kendall, 1990; Weisz et al. 1997). Both Coping Cat and PASCET have been used in small studies of children with physical illnesses (Blocher et al. 2013; Szigethy et al. 2007). The use and order of the modules within therapy is guided by an algorithm.

MATCH-ADTC is designed to account for the high rates of co-morbidities in usual clinical practice and has been demonstrated to be superior to usual care in children with common mental health disorders (Weisz et al. 2012). According to the protocol, the therapist focuses on the initial problem area identified as most important, based on measures and patient priorities. It is considered to represent true evidence-based practice in that the protocol combines research evidence, clinical judgement and patient values and preferences. Once a problem area has been selected (e.g. anxiety), an algorithm specifies a default sequence of modules 
and guides clinical judgement. However, the intervention is personalised so that if the default sequence cannot be implemented (e.g. due to low mood), then the sequence can be changed to address the immediate issue. Once that issue has been addressed, treatment for the original problem area is resumed.

This intervention seemed particularly suitable for our needs considering the high rates of co-morbidities or co-occurring mental health difficulties in our population of young people with epilepsy (Reilly et al. 2014). We wanted the intervention to maximize accessibility for an under-treated population and decided to implement a stepped-care approach, through offering MATCH-ADTC as a guided self-help intervention. Following this, we could refer onto higher intensity treatments if necessary. This is the first time that MATCH-ADTC has been used in a guided self-help format.

The intervention took the form of a ten-session guided self-help therapy based on the MATCH-ADTC protocol. One-month and three-month follow-up calls were also completed. Each telephone call involved setting an agenda, reviewing the homework, problem solving any difficulties, setting the next week's homework and summarising the session. As a guided selfhelp intervention, no new material was 'taught' during the sessions. Instead, the homework consisted of the therapist emailing a relevant strategy to the participant, which they would read, attempt to implement and then record the outcome. The purpose of the telephone calls was to problem-solve any difficulties in implementation and enhance motivation. The therapist was also able to use the MATCH algorithm to ensure that the correct module was being followed each week. The therapists (R.S. and S.B.) were both qualified clinical psychologists.

\section{Measures}

Measures were completed in line with the Children and Young People's Improving Access to Psychological Therapies (CYPIAPT) measurement protocol, to allow comparison to national data sets. Both pre-post measures and weekly measures were completed.

\section{Diagnostic}

The Development and Wellbeing Assessment (DAWBA; Goodman et al., 2000) - a package of interviews, questionnaires and rating techniques designed to generate ICD-10 and DSM-5 psychiatric diagnoses on 5- to 17-year-olds. It takes 30-50 minutes to complete in a community sample. It is used widely in child and adolescent mental health services internationally and has been demonstrated to be acceptable to families attending child and adolescent mental health services (Last et al. 2013). There are a variety of formats including computer-administered interviews that are available via the internet in a number of languages. The online version provides probabilities of a child meeting diagnostic criteria and can additionally be clinician rated for the presence or absence of diagnoses. It demonstrates good psychometric properties (Cohen's kappa $=74-99 \%$ for specific diagnoses when comparing rated DAWBA diagnoses and clinicians' diagnoses).

\section{Pre, post and follow-up measures}

Strengths and Difficulties Questionnaire (SDQ; Goodman, 1997) - the SDQ is a commonly used and psychometrically robust measure to identify emotional and behavioural symptoms. 
It consists of 25 items, divided into five scales (emotional symptoms, conduct problems, hyperactivity/inattention, peer relationship problems), in addition to an impact supplement which asks the respondent whether they think the young person has an emotional/behavioural problem, and if so asks about chronicity, distress, social impairment and burden to others. Scores on each subscale and the impact scale can be categorised into four ranges: close to average, slightly high, high, and very high. It can be completed by the parent if the child is younger than 11 years of age, or by the parent and young person if they are age 11 or older. It has demonstrated high sensitivity and specificity in predicting psychiatric diagnoses in children with chronic physical illness (Hysing et al. 2007) and has been suggested for use as a 'clinic-friendly' screening tool for identifying mental health problems in children with epilepsy (Asato et al. 2015).

Revised Child Anxiety and Depression Scale (RCADS; Chorpita et al. 2000) - this is a wellvalidated 47-item parent or self-report questionnaire identifying symptoms of depression and anxiety in children and young people.

Paediatric Quality of Life (PedsQL; Varni et al. 2001) - this is a 23-item well-validated parent or self-report measure of quality of life with four subscales, covering physical, emotional, social and school functioning.

\section{Weekly measures}

Symptom monitoring - 'How are things? Depression' - this is a weekly self-report questionnaire which identifies symptoms of depression. It is an 8-item scale, based on the depression subscale of the RCADS.

Goal Based Outcomes - Chloe identified three goals for treatment at the initial appointment. Progress towards the goals is rated on a scale of 1-10 in each session (where 1 is no progress towards goals, and 10 is goal is met).

\section{Procedures}

Participants were recruited from a related study investigating routine detection of mental health disorders in children and young people attending neurology clinics. All children/young people within the correct age range attending neurology clinics were sent information leaflets about the study prior to attending the clinic. When in clinic, they were approached by a research assistant, who invited the parents/carers, and young person where appropriate, to complete the Strengths and Difficulties Questionnaire online on a tablet computer within the clinic. Informed consent was given for the full study at this point. Those scoring above a pre-specified threshold (defined as the combination of raised symptom score: $\geq 14$ out of a maximum of 40, which is a score in the 'slightly raised' range or higher, and raised impact score: $\geq 2$ out of a maximum of 10 indicating a score in the 'high' range or above) were automatically invited by the system to progress to the full Development and Wellbeing Assessment, which was completed by the parent/carer and young person where appropriate at home. The DAWBA was blind rated by a clinician not involved in the study intervention.

\section{Relevant background history}

Chloe had severe intractable epilepsy, with multiple daily seizures. Due to the severity of the epilepsy, she had undergone surgery a year prior to our assessment, involving removal of the 
brain area that was thought to be the focal point of the seizures (the part of the brain from which the seizures arose). Whilst this carried significant risks, there was a high likelihood of Chloe becoming seizure free following the operation. Unfortunately, Chloe had experienced some seizures following the surgery, although these were far less frequent. One of these seizures led to Chloe falling suddenly and hurting herself.

All patients who are contemplating epilepsy surgery are offered neuropsychological and neuropsychiatric assessments prior to and after the surgery. The neuropsychiatrist was alerted to Chloe's deteriorating mood shortly following the surgery after her parents telephoned the service. Chloe had been feeling particularly low since the surgery, as it had not resulted in seizure freedom as she had hoped. Her parents reported that she was very upset and angry, although was reluctant to talk to them about her feelings. Chloe had told them that that she 'might as well be dead'. She also had some difficulty sleeping, which can occur following neurosurgery. Chloe was therefore referred to her local CAMHS for review at this point.

Chloe and her parents were then offered a neuropsychiatric appointment 10 weeks postoperatively. Chloe and her parents both reported that she was unusually irritable, sensitive and distressed. She was tearful on most days of the week, and was sensitive to perceived injustices or criticisms. There was also some conflict between Chloe and her parents, as they adjusted to Chloe having far fewer seizures, whilst managing the uncertainty of possible seizures and Chloe's increasing independence as she moved towards adulthood. Chloe felt that her parents restricted her activities because of the concern that being out late or staying away from home could increase the risk of a seizure. Chloe wanted some suggestions for thinking about low mood and anxiety. At this point, the psychiatrist felt that it might be helpful for the family to have some joint sessions to discuss how to negotiate her independence in the context of the epilepsy. However, Chloe did not want 'family therapy' and the psychiatrist gave the family time to think about this and to contact them or community CAMHS if they did decide that they wanted referral for further input.

\section{Assessment interview}

The DAWBA rated the overall possibility of a disorder as 'high'. Predicted probability of generalized anxiety and eating disorder were calculated at around 50\%, and for obsessive compulsive disorder, depression and hyperactivity around 3\%, although the clinician rating suggested that she did not meet full diagnostic criteria for any disorder. The open-ended text comments suggested that Chloe was experiencing some anxiety, low mood and had worries regarding her eating, which appeared to have been exacerbated by the recent neurosurgery.

On this basis, Chloe met criteria for face-to-face assessment with a view to intervention as part of the research study.

We completed our assessment approximately four months following the last contact that Chloe had had with the neuropsychiatry service. The full assessment was a combination of a computerized diagnostic interview (DAWBA; Goodman et al. 2000) and goal-based, to determine eligibility for the research study intervention.

Chloe described spending a lot of time comparing herself with other people, such as her older siblings and best friend. She often thought that she did not compare favourably with them, and worried about her appearance, weight and academic abilities, as well as the impact of epilepsy on her life. Chloe had missed a significant amount of school due to the epilepsy surgery, and felt behind academically compared with peers and family. She reported that her mood was very labile and that she frequently became tearful. For example, she said that she 
might be very happy all day but if someone said something that upset her, she would become very tearful and low. Chloe's self-evaluation was overly dependent on her eating, shape and weight. She tried to limit her calorie intake and wanted to have more control over the intake of food, although she did eat three meals per day. Objectively, she was in the healthy weight range, but her concerns about eating were that if she put on more weight, then people would not like her. She worried about her appetite and weight; she explained that she used food as comfort, but did want to lose weight. She was highly pre-occupied by thoughts of eating, shape and weight and such pre-occupation interfered with her daily life, for example her ability to concentrate on her work. She also reported poor sleep, saying that it took her two hours to get to sleep. She thought that this may be partly due to the neurosurgery and medications. At the time of the assessment, she was taking additional medications to help her sleep. Her mood was lower when she had not slept well. Chloe made an effort to see her friends frequently, which she had noticed had a positive impact on her mood.

Regarding goals, Chloe wanted to work on:

1. Reducing comparison with other people;

2. Being able to get to sleep more quickly;

3. Worrying less about what she ate.

\section{Formulation and plan}

Chloe had symptoms of depression, anxiety and an unspecified eating disorder. Whilst these did not meet full diagnostic criteria, they were significantly impairing, and causing difficulties in day-to-day life. Angold and colleagues (1999) demonstrated that in children and young people, 'across a variety of "caseness measures", those with symptomatic impairment proved to be more disturbed than those without either a diagnosis or impairment, and as disturbed as those with a diagnosis but without impairment'. We therefore considered that it was important to offer intervention for these impairing symptoms.

Many of the symptoms stemmed from beliefs around not being good enough, either academically or in appearance, in comparison with peers and high-achieving family members, and worry about the future. Her symptoms were linked to epilepsy and the impact that this had had on her cognitive abilities, education, and her future as an independent young woman.

The intervention investigated in the research study was a modular intervention, which was chosen as it could address multiple co-occurring common mental health problems, i.e. anxiety, depression and disruptive behaviour. In Chloe's case, we had to make the clinical decision as to whether to begin the treatment with the anxiety module or the depression module, given these two areas of difficulties were identified by Chloe as priorities. Chloe's goals for treatment were broad and could be seen as relating to depression, anxiety, eating and low self-esteem, but we jointly decided with Chloe to start with the depression module, for several reasons. Firstly, Chloe thought that all of the worries were secondary to her present low mood. Secondly, the anxiety module is based on evidence-based protocols for the treatment of anxiety in children. These are largely behaviourally based, involving hierarchies and gradual exposure to situations that make the child or young person anxious. Chloe was not avoiding any activities and her anxiety was primarily cognitively based worry linked to low self-esteem. Finally, Chloe was tearful on presentation and low in motivation and we were concerned that 
low mood would interfere with treatment progress for anxiety. These reasons, together with the known high risks of suicide in people with epilepsy (e.g. Christensen et al., 2007) led us to focus initially on treatment of the low mood while simultaneously monitoring her anxiety and eating difficulties.

Chloe was therefore offered a ten-session telephone guided self-help intervention as part of the research study, using a depression protocol focused on her goals for treatment in the first instance. The flexibility of the modular approach allowed us to potentially change to the anxiety module if this was necessary.

Due to her age and personal preferences, all sessions were undertaken with Chloe and without involvement of her parents.

Materials sent after each session were those included in most evidence-based protocols for the treatment of depression (Table 1).

\section{Outcome}

\section{Weekly measures}

Figures 1 and 2 illustrate progress towards goals and ratings on the symptom based measure, respectively. They demonstrate that progress was made towards goals, and symptoms decreased across treatment. There was some understandable fluctuation that was linked to life events, such as an EEG demonstrating some remaining epileptic activity.

\section{Pre, post and follow-up measures}

\section{$S D Q$}

Whilst the overall stress scale of the SDQ did not demonstrate clinically significant change for either the parent or young person measures, the parent-reported emotional subscale score was 7 prior to intervention (very high range), 5 post-intervention and at 1-month follow-up (high range), and 3 at the 3-month follow-up (average range) (see Table 2). The young person reported score was 7 prior to intervention (very high range), reducing to 3 (average range) at post-intervention and 1-month follow-up time points, and 4 (slightly raised) at the 3-month follow-up. There was clinically significant and reliable change on the young person report between pre-intervention and post-intervention measures, but not between pre-intervention and 3-month follow-up.

\section{RCADS}

The parent reported RCADS total Anxiety and Depression scores fell within the clinical range ( $\mathrm{T}$ scores above 70 ) prior to intervention and within the non-clinical range post-intervention. It remained in the non-clinical range at both follow-up time points. On examining the anxiety and depression scales separately, the change was mostly within the anxiety scales, and not depression. The young person reported RCADS total anxiety and depression scores fell within the non-clinical range at all time points. 
Table 1. Content of intervention sessions

\begin{tabular}{|c|c|c|}
\hline Session & Topic & Additional information \\
\hline 1 & Goal setting & $\begin{array}{l}\text { We confirmed Chloe's goals outlined in the } \\
\text { face-to-face assessment }\end{array}$ \\
\hline 2 & $\begin{array}{l}\text { Psychoeducation - 'getting } \\
\text { acquainted with } \\
\text { depression and the CBT } \\
\text { model' }\end{array}$ & $\begin{array}{l}\text { Chloe found the psycho-education sheets } \\
\text { particularly helpful, as she realised that her } \\
\text { mood could be affected by her thoughts, and } \\
\text { that her thoughts were not necessarily 'the } \\
\text { truth'. This worked for a range of difficulties, } \\
\text { from thoughts around her inadequacy to her } \\
\text { worries about eating }\end{array}$ \\
\hline 3 & Problem solving & $\begin{array}{l}\text { Chloe had some difficulties with family } \\
\text { members and social situations, and } \\
\text { problem-solving strategies were particularly } \\
\text { helpful in managing these. One problem was } \\
\text { that Chloe had a slightly increased risk of } \\
\text { seizures if she stayed out late at night and did } \\
\text { not get much sleep. Her parents were } \\
\text { understandably concerned about this and so } \\
\text { Chloe often had to come home early, which } \\
\text { increased her thoughts of being different and } \\
\text { inadequate. Through problem solving, she } \\
\text { was able to find solutions to this, including } \\
\text { getting a medical opinion as to the risks of } \\
\text { staying up late, and negotiating late nights } \\
\text { with her parents. With this additional } \\
\text { information, as well as the psycho-education } \\
\text { sheets, she was also able to see that her } \\
\text { thought that she was not sleeping well enough } \\
\text { was actually worry about sleep; she was in } \\
\text { fact sleeping a normal amount }\end{array}$ \\
\hline 4 & $\begin{array}{l}\text { Behavioural activation - } \\
\text { 'activity selection' }\end{array}$ & $\begin{array}{l}\text { Chloe had avoided going out with some friends } \\
\text { as she felt inadequate in comparison with } \\
\text { them. Using the new thinking strategies that } \\
\text { she had learnt in the psycho-education sheets, } \\
\text { she was able to overcome her worries of } \\
\text { inadequacy, and so started to plan more } \\
\text { activities in which she noticed resulted in an } \\
\text { improvement in mood. As she exposed } \\
\text { herself to more of these situations, she was } \\
\text { able to test out more of her cognitions of } \\
\text { inadequacy. She also found that increasing } \\
\text { her activity level through sports, and watching } \\
\text { films, had a positive impact on her mood }\end{array}$ \\
\hline 5 & $\begin{array}{l}\text { Relaxation - 'learning to } \\
\text { relax' }\end{array}$ & $\begin{array}{l}\text { Chloe found simple relaxation strategies } \\
\text { helpful, particularly following difficult } \\
\text { encounters with friends or family }\end{array}$ \\
\hline
\end{tabular}


Table 1. Continued

\begin{tabular}{|c|c|c|}
\hline Session & Topic & Additional information \\
\hline 6 & $\begin{array}{l}\text { Relaxation - 'quick } \\
\text { calming' }\end{array}$ & $\begin{array}{l}\text { These sheets followed on from the learning to } \\
\text { relax strategies }\end{array}$ \\
\hline 7 & $\begin{array}{l}\text { Social skills - 'presenting a } \\
\text { positive self' }\end{array}$ & $\begin{array}{l}\text { Chloe learnt that presenting herself positively } \\
\text { and confidently led to people responding to } \\
\text { her positively and confidently }\end{array}$ \\
\hline 8 & $\begin{array}{l}\text { Cognitive strategies - } \\
\text { 'Cognitive Coping - } \\
\text { BLUE' }\end{array}$ & $\begin{array}{l}\text { These strategies reinforced the cognitive } \\
\text { strategies that Chloe learnt in the } \\
\text { psycho-education. Chloe continued to find } \\
\text { these cognitive aspects particularly helpful }\end{array}$ \\
\hline 9 & $\begin{array}{l}\text { Cognitive strategies - } \\
\text { 'Cognitive coping - TLC' }\end{array}$ & $\begin{array}{l}\text { These strategies combined the relaxation and } \\
\text { cognitive strategies to use in difficult } \\
\text { situations }\end{array}$ \\
\hline 10 & $\begin{array}{l}\text { Relapse prevention - } \\
\text { 'Plans for coping' }\end{array}$ & $\begin{array}{l}\text { Chloe completed a relapse prevention plan } \\
\text { prior to the final session }\end{array}$ \\
\hline $\begin{array}{l}\text { Follow-up } \\
\text { sessions: } 1 \text { month } \\
\text { and } 3 \text { months }\end{array}$ & & $\begin{array}{l}\text { Strategies were reinforced at both follow-up } \\
\text { time points }\end{array}$ \\
\hline
\end{tabular}

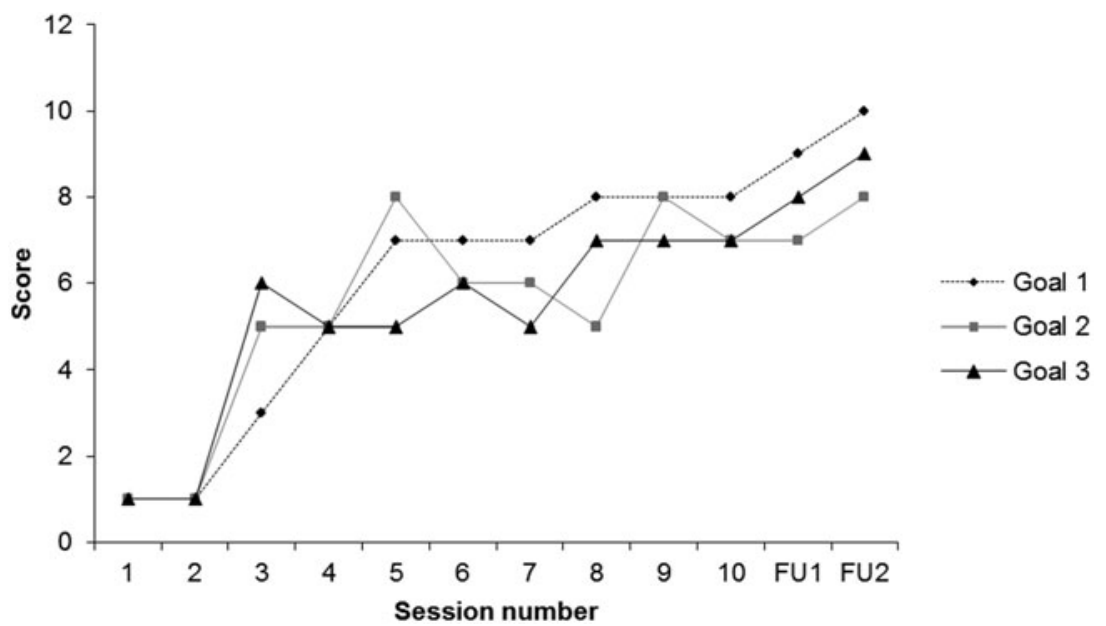

Fig. 1. Client-reported session-by-session progress towards goals on a 1-10 scale

\section{PedsQL}

Total PedsQL scores were in the clinical range prior to intervention, and fell within the nonclinical range post-intervention and at first follow-up. These changes were not stable, however. The scores then reduced to the clinical range by 3-month follow-up. 


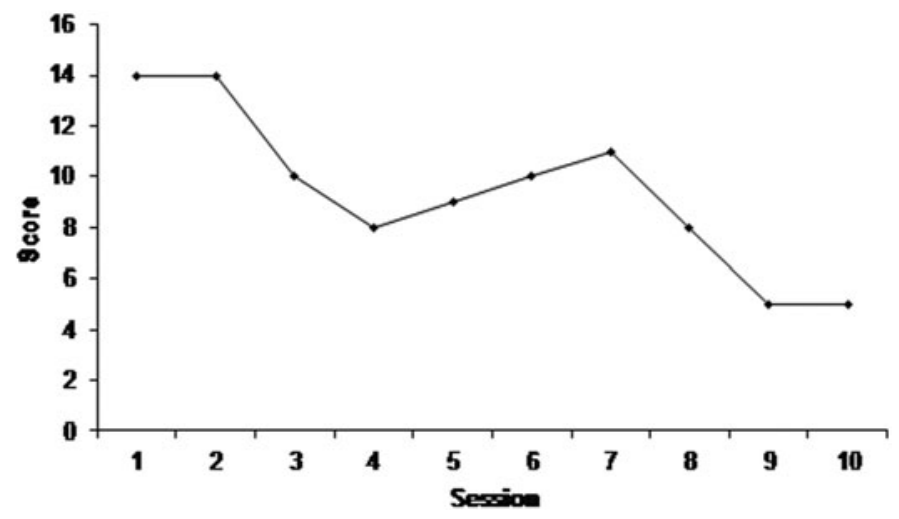

Fig. 2. Client-reported session-by-session progress on the depression symptom measure: 'How are things? Depression'

\section{Other outcomes}

Chloe was interviewed about her experience of the intervention by a research assistant who was not involved in the intervention delivery. She and her parents also completed an Experience of Service Questionnaire. Chloe said that she found the intervention helpful, that she liked the convenience of having the therapy over the telephone, and found that it was able to fit around her life more easily. She also said that she found it easier to talk to someone on the telephone rather than in person. Chloe's eating difficulties had been informally monitored throughout treatment and Chloe reported that they were much improved post-treatment and at follow-up. At the end of treatment and at follow-up, Chloe did not meet criteria for any Axis 1 disorder as assessed by clinician rated DAWBA.

\section{Discussion}

This 'simple' intervention appeared to be effective in working towards Chloe's goals in this 'complex' case (notwithstanding limitations of single case designs and measurement). The standard strategies were able to tackle epilepsy-related difficulties (e.g. worry about having another seizure) as well as those that seemed more distantly linked to the epilepsy (such as worries about weight). The intervention used was in fact simpler than we had originally anticipated. The intervention was based on the MATCH-ADTC protocol because of its modular nature, which allows us to treat co-occurring mental health problems through use of different modules. We did not have to switch modules, as progression was maintained throughout treatment. This meant that in reality, we had used a guided self-help version of an evidence-based treatment for depression.

The meaning of this is unclear and comes back to the issue of what defines a complex case. Clinically, this presentation is neurologically complex, but very straightforward from a mental health point of view. It seems as though the co-morbid chronic illness may cloud judgement of what is in fact a straightforward presentation of depression and anxiety in a young person, which evidence suggests can be treated using guided self-help (Pennant et al. 2015). It seems that complex cases are self-defining as those which do not respond well to standard treatments 
Table 2. Scores on standardized measures at pre-and post-intervention and 1- and 3-month follow-up sessions

\begin{tabular}{|c|c|c|c|c|}
\hline Scale & $\begin{array}{l}\text { Score pre- } \\
\text { intervention }\end{array}$ & $\begin{array}{l}\text { Score post- } \\
\text { intervention }\end{array}$ & $\begin{array}{l}\text { Score at } \\
\text { 1-month } \\
\text { follow-up }\end{array}$ & $\begin{array}{l}\text { Score at } \\
\text { 3-month } \\
\text { follow-up }\end{array}$ \\
\hline $\begin{array}{l}\text { SDQ parent report } \\
\text { overall stress }\end{array}$ & $\begin{array}{l}16 \text { (slightly } \\
\text { raised) }\end{array}$ & $\begin{array}{l}15 \text { (slightly } \\
\text { raised) }\end{array}$ & 17 (high) & $\begin{array}{l}16 \text { (slightly } \\
\text { raised) }\end{array}$ \\
\hline $\begin{array}{l}\text { SDQ young person } \\
\text { report overall } \\
\text { stress }\end{array}$ & 19 (high) & $\begin{array}{l}16 \text { (slightly } \\
\text { raised) }\end{array}$ & $\begin{array}{l}16 \text { (slightly } \\
\text { raised) }\end{array}$ & 18(high) \\
\hline $\begin{array}{l}\text { RCADS parent } \\
\text { report total } \\
\text { Anxiety and } \\
\text { Depression ( } \mathrm{T} \\
\text { score) }\end{array}$ & $54(74)$ & $34(59)^{*+}$ & $34(59)^{*+}$ & $41(64)^{*+}$ \\
\hline $\begin{array}{l}\text { RCADS young } \\
\text { person report total } \\
\text { Anxiety and } \\
\text { Depression }\end{array}$ & $34(44)$ & $22(38)$ & $28(41)$ & $26(4)$ \\
\hline $\begin{array}{l}\text { PedsQL parent } \\
\text { report total }\end{array}$ & 56.52 & $66.3^{*}$ & $75^{*}$ & 61.25 \\
\hline $\begin{array}{l}\text { PedsQL young } \\
\text { person report total }\end{array}$ & 66.3 & $73.91^{*}$ & $73.91^{*}$ & 65.22 \\
\hline
\end{tabular}

*Clinically significant change between pre-intervention score and post score, 1-month follow-up or 3month follow-up; ${ }^{+}$reliable change between pre-intervention score and post score, 1-month follow-up or 3-month follow-up (for changes which were clinically significant).

and therefore may require a different and/or more personalised approach. However, this case report provides some evidence that until we attempt to treat them, we do not know whether or not they will respond to an intervention. To date, it appears that services (and perhaps clients themselves) may have assumed that the mental health problems of children and young people with chronic illnesses are complex and will not respond to intervention, despite no clear evidence of this.

Epstein (1984) defined a 'modern principle of parsimony' for psychology as 'where we have no reason to do otherwise and where two theories account for the same facts, we should prefer the one which is briefer, which makes assumptions with which we can easily dispense, which refers to observables, and which has the greatest possible generality'. In this case, we have two alternative 'theories'; the first, that mental health disorders in the presence of chronic physical illness create complexity such that standard interventions, known to be efficacious across a wide range of populations, will not be effective; the second, that these cases are no more complex than those seen within usual clinical practice. Following the principle of parsimony, we would suggest that until there is evidence to suggest that children and young people with mental health difficulties in the context of chronic illness are not treatable via the current evidence-based interventions for mental health difficulties in children and young people, that 
research is focused on determining whether they are treatable using these methods, in order to maximise access to intervention as quickly as possible to an undertreated group.

\section{Contribution to the literature}

Evidence-based CBT interventions have a very small evidence base in treating mental health disorders in children and young people with chronic illness. Whilst there is a wide evidencebase for paediatric psychology interventions more broadly, very few studies investigate samples where patients meet pre-defined criteria on a measure of mental health (Bennett et al. 2015). This case report is very preliminary evidence that it may be possible to treat mental health problems in the context of chronic neurological conditions in children using a guided self-help approach. It is also the first study to use the MATCH-ADTC intervention in a guided self-help format.

\section{Limitations}

This case report may provide preliminary evidence to suggest that that it is possible to use simple guided self-help CBT interventions to treat mental health disorders in the context of apparent complexity due to a chronic illness. However, there are a number of limitations to this conclusion. Firstly, whilst it is debatable whether the physical illness adds to the complexity of the case overall, it does add a level of complexity to measurement; for example, the quality of life measure is directly impacted by physical health. Similarly, other measures, although not measuring physical health per se, are likely to be affected by physical health. For example, some questions are directly associated with physical symptoms (on the SDQ, one item is 'Often complains of headaches, stomach aches or sickness'), or may be impacted by external events at any given time point (e.g. 'Many worries, often seems worried'). We know that at the time of the second follow-up, Chloe was experiencing worries because of results of a recent EEG suggesting possible remaining epileptic activity, and this may be one reason that the Quality of Life measure reverted back to the clinical range at 3-month follow-up. However, Chloe was able to cope with these worries, and her progress towards the specific goals outlined at the start of the intervention was maintained. There were several other measurement issues within the case, including discrepancies between parent and young person report on the measures. It raises questions regarding whether broad symptom based measures are the best measure of progress in these cases, or whether in fact specific goalbased measures may be more reflective of change within therapy.

Secondly, the nature of the study design, involving only one participant, and without repeated baseline measures, is limited. It may simply have been the passage of time, over which Chloe gradually adapted to life and transitioning into a young adult. Further research is needed to investigate the use of standard CBT interventions (included guided self-help) for the treatment of mental health disorders in children and young people with chronic neurological conditions.

\section{Main points}

(1) This case report is initial evidence that it may be possible to use simple guided self-help CBT interventions to treat mental health disorders in the context of apparent complexity due to a chronic illness. 
(2) Given that clinicians consider a large proportion of their cases to be complex, it is not feasible to offer all patients 'complex' interventions and it may not be necessary.

(3) Guided self-help appears to be a clinically and cost-effective way to disseminate evidencebased practice to a large number of people and further research is needed to determine whether this is the case for young people with mental health difficulties in the context of chronic illness.

\section{Recommended follow-up reading}

Borntrager CF, Chorpita BF, Higa-McMillan C, Weisz JR (2015). Provider attitudes toward evidence-based practices: are the concerns with the evidence or with the manuals? Psychiatric Services 60, 677-681.

Chorpita BF, Weisz JR (2009). Modular approach to therapy for children with anxiety, depression, trauma, or conduct problems (MATCH-ADTC). Satellite Beach, FL: PracticeWise LLC.

Weisz JR, Chorpita BF, Palinkas LA, Schoenwald SK, Miranda J, Bearman SK et al. (2012). Testing standard and modular designs for psychotherapy treating depression, anxiety, and conduct problems in youth: A randomized effectiveness trial. Archives of General Psychiatry 69, 274-282.

\section{References}

Angold A, Costello EJ, Farmer EM, Burns BJ, Erkanli A (1999). Impaired but undiagnosed. Journal of the American Academy of Child \& Adolescent Psychiatry 38, 129-137.

Asato MR, Doss JL, Plioplys S (2015). Clinic-friendly screening for cognitive and mental health problems in school-aged youth with epilepsy. Epilepsy \& Behavior 48, 97-102.

Barkley RA (1997). Defiant Children: A Clinician's Manual for Assessment and Parent Training (2nd edition). New York, NY: Guilford Press.

Bennett S, Shafran R, Coughtrey A, Walker S, Heyman I (2015). Psychological interventions for mental health disorders in children with chronic physical illness: a systematic review. Archives of Disease in Childhood 100, 308-316.

Blocher JB, Fujikawa M, Sung C, Jackson DC, Jones JE (2013). Computer-assisted cognitive behavioral therapy for children with epilepsy and anxiety: a pilot study. Epilepsy and Behavior 27, 70-76.

Children's Commissioner (2016). Lightning Review: Access to Child and Adolesent Mental Health Services May 2016. Available at: https://www.childrenscommissioner.gov.uk/publication/ lightning-review-access-to-child-and-adolescent-mental-health-services/

Chorpita BF, Weisz JR (2009). Modular approach to therapy for children with anxiety, depression, trauma, or conduct problems (MATCH-ADTC). Satellite Beach, FL: PracticeWise LLC.

Chorpita BF, Yim L, Moffitt C, Umemoto LA, Francis SE (2000). Assessment of symptoms of DSM-IV anxiety and depression in children: A revised child anxiety and depression scale. Behaviour Research and Therapy 38, 835-855.

Christensen J, Vestergaard M, Mortensen PB, Sidenius P, Agerbo E (2007). Epilepsy and risk of suicide: a population-based case-control study. The Lancet Neurology 6, 693-698.

Clarke DF, Roberts W, Daraksan M, Dupuis A, McCabe J, Wood H et al. (2005). The prevalence of autistic spectrum disorder in children surveyed in a tertiary care epilepsy clinic. Epilepsia 46, 1970-1977.

Cottrell D (2015). Prevention and treatment of psychiatric disorders in children with chronic physical illness. Archives of Disease in Childhood 100, 303-304. 
Cuijpers P, Donker T, van Straten A, Li J, Andersson G (2010). Is guided self-help as effective as face-to-face psychotherapy for depression and anxiety disorders? A systematic review and metaanalysis of comparative outcome studies. Psychological Medicine 40, 1943-1957.

Dunn DW, Austin JK, Harezlak J (2003). ADHD and epilepsy in childhood. Developmental Medicine and Child Neurology 45, 50-54.

Epstein R (1984). The principle of parsimony and some applications in psychology. The Journal of Mind and Behavior 5, 119-130.

Ettinger AB (2006). Psychotropic effects of antiepileptic drugs. Neurology 67, 1916-1925.

Fastenau PS, Shen J, Dunn DW, Austin JK (2008). Academic underachievement among children with epilepsy proportion exceeding psychometric criteria for learning disability and associated risk factors. Journal of Learning Disabilities 41, 195-207.

Goodman R (1997). The Strengths and Difficulties Questionnaire: a research note. Journal of Child Psychology and Psychiatry 38, 581-586.

Goodman R, Ford T, Richards H, Gatward R, Meltzer H (2000). The Development and Well-Being Assessment: description and initial validation of an integrated assessment of child and adolescent psychopathology. Journal of Child Psychology and Psychiatry 41, 645-655.

Hadert A (2013). Adapting Cognitive Behavioural Therapy Interventions for Anxiety or Depression to Meet the Needs of People with Long-term Physical Health Conditions: A Mixed-Methods Study (Doctoral dissertation). Retrieved from: http://hdl.handle.net/10871/13705

Hesdorffer DC, Ishihara L, Mynepalli L, Webb DJ, Weil J, Hauser WA (2012). Epilepsy, suicidality, and psychiatric disorders: a bidirectional association. Annals of Neurology 72, 184-191.

Heyman I, Skuse D, Goodman R (2015a). Brain disorders and psychopathology. In A Thapar, DS Pine, JF Leckman, S Scott, MJ Snowling, E Taylor (eds). Rutter's Child and Adolescent Psychiatry, 6th edition, pp. 389-402. Chichester, UK: John Wiley \& Sons.

Hofmann SG, Asnaani A, Vonk IJ, Sawyer AT, Fang A (2012). The efficacy of cognitive behavioral therapy: a review of meta-analyses. Cognitive Therapy and Research 36, 427-440.

Hoppe C, Elger CE (2011). Depression in epilepsy: a critical review from a clinical perspective. Nature Reviews Neurology 7, 462-472.

Huhn M, Tardy M, Spineli LM, Kissling W, Förstl H, Pitschel-Walz G et al. (2014). Efficacy of pharmacotherapy and psychotherapy for adult psychiatric disorders: a systematic overview of metaanalyses. Journal of the American Medical Association Psychiatry 71, 706-715.

Hysing M, Elgen I, Gillberg C, Lie SA, Lundervold AJ (2007). Chronic physical illness and mental health in children. Results from a large-scale population study. Journal of Child Psychology and Psychiatry 48, 785-792.

James AD, James G, Cowdrey FA, Soler A, Weatherall R (2005). Cognitive behavioural therapy for anxiety disorders in children and adolescents. Cochrane Database of Systematic Reviews $\mathbf{4}$, CD004690.

Kanner AM (2011). Depression and epilepsy: a bidirectional relation? Epilepsia 52 (S1), 21-27.

Kanner AM (2003). Depression in epilepsy: prevalence, clinical semiology, pathogenic mechanisms, and treatment. Biological Psychiatry 54, 388-398.

Katon WJ (2003). Clinical and health services relationships between major depression, depressive symptoms, and general medical illness. Biological Psychiatry 54, 216-226.

Katon WJ, Von Korff M, Lin EH, Simon G, Ludman E, Russo J et al. (2004). The pathways study: a randomized trial of collaborative care in patients with diabetes and depression. Archives of General Psychiatry 61, 1042-1049.

Katon WJ, Richardson L, Russo J, Lozano P, McCauley E (2006). Quality of mental health care for youth with asthma and comorbid anxiety and depression. Medical Care 44, 1064-1072.

Kendall PC (1990). Coping Cat Manual. Ardmore, PA: Workbook Publishing.

Kovacs M, Mukerji P, Drash A, Iyengar S (1995). Biomedical and psychiatric risk factors for retinopathy among children with IDDM. Diabetes Care 18, 1592-1599. 
Last A, Ford T, Henley W, Norman S, Goodman R (2013). Feasibility of the Development and Well Being Assessment as an adjunct to clinical assessment in child and adolescent mental health services. Child and Adolescent Mental Health 19, 142-146.

Lau AS (2006). Making the case for selective and directed cultural adaptations of evidence-based treatments: examples from parent training. Clinical Psychology: Science and Practice 13, 295310.

Lespérance F, Frasure-Smith N, Talajic M, Bourassa MG (2002). Five-year risk of cardiac mortality in relation to initial severity and one-year changes in depression symptoms after myocardial infarction. Circulation 105, 1049-1053.

Lilienfeld SO, Ritschel LA, Lynn SJ, Cautin RL, Latzman RD (2013). Why many clinical psychologists are resistant to evidence-based practice: root causes and constructive remedies. Clinical Psychology Review 33, 883-900.

Lousada J, Weisz J, Hudson P, Swain T (2015). Psychotherapy provision in the UK: time to think again. The Lancet Psychiatry 2, 289-291.

McGrady ME, Hood KK (2013). Cognitive behavioral therapy for adolescents with Type 1 diabetes and subclinical depressive symptoms. Diabetes Management 3, 207-215.

Nadkarni S, Devinsky O (2005). Psychotropic effects of antiepileptic drugs. Epilepsy Currents 5, 176181.

NICE (2005). Depression in children and young people: identification and management. Available at:http://www.nice.org.uk/CG28

NICE (2009a). Depression in adults: recognition and management (last updated 2016). Available at: http://www.nice.org.uk/CG090

NICE (2009b). Treatment and management of depression in adults, including adults with a chronic physical health problem. Available at: http://www.nice.org.uk/CG091

NICE (2011). Generalised anxiety disorder and panic disorder in adults: management. Available at:https://www.nice.org.uk/guidance/cg113

Ott D, Siddarth P, Gurbani S, Koh S, Tournay A, Shields WD, Caplan R (2003). Behavioral disorders in pediatric epilepsy: unmet psychiatric need. Epilepsia 44, 591-597.

Pennant ME, Loucas CE, Whittington C, Creswell C, Fonagy P, Fuggle P et al. (2015). Computerised therapies for anxiety and depression in children and young people: a systematic review and meta-analysis. Behaviour Research and Therapy 67, 1-18.

Prince M, Patel V, Saxena S, Maj M, Maselko J, Phillips MR, Rahman A (2007). No health without mental health. The Lancet 370, 859-877.

Reigada LC, Benkov KJ, Bruzzese JM, Hoogendoorn C, Szigethy E, Briggie A et al. (2013). Integrating illness concerns into cognitive behavioral therapy for children and adolescents with inflammatory bowel disease and co-occurring anxiety. Journal for Specialists in Pediatric Nursing 18, 133-143.

Reilly C, Atkinson P, Das KB, Chin RF, Aylett SE, Burch V et al. (2014). Neurobehavioral comorbidities in children with active epilepsy: a population-based study. Pediatrics 133, e1586e1593.

Shafran R, Clark DM, Fairburn CG, Arntz A, Barlow DH, Ehlers A et al. (2009). Mind the gap: improving the dissemination of CBT. Behaviour Research and Therapy 47, 902-909.

Sillanpää M (2004). Learning disability: occurrence and long-term consequences in childhood-onset epilepsy. Epilepsy and Behavior 5, 937-944.

Stirman SW, Crits-Christoph P, DeRubeis RJ (2004). Achieving successful dissemination of empirically supported psychotherapies: a synthesis of dissemination theory. Clinical Psychology: Science and Practice 11, 343-359.

Szigethy E, Kenney E, Carpenter J, Hardy DM, Fairclough D, Bousvaros A et al. (2007). Cognitivebehavioral therapy for adolescents with inflammatory bowel disease and subsyndromal depression. Journal of the American Academy of Child and Adolescent Psychiatry 46, 1290-1298. 
Tellez-Zenteno JF, Patten SB, Jetté N, Williams J, Wiebe S (2007). Psychiatric comorbidity in epilepsy: a population-based analysis. Epilepsia 48, 2336-2344.

Varni JW, Seid M, Kurtin PS (2001). PedsQL ${ }^{\mathrm{TM}}$ 4.0: Reliability and validity of the Pediatric Quality of Life Inventory ${ }^{\mathrm{TM}}$ Version 4.0 Generic Core Scales in healthy and patient populations. Medical Care 39, 800-812.

Weisz JR, Chorpita BF, Palinkas LA, Schoenwald SK, Miranda J, Bearman SK et al. (2012). Testing standard and modular designs for psychotherapy treating depression, anxiety, and conduct problems in youth: a randomized effectiveness trial. Archives of General Psychiatry 69, 274-282.

Weisz JR, Thurber CA, Sweeney L, Proffitt VD, LeGagnoux GL (1997). Brief treatment of mildto-moderate child depression using primary and secondary control enhancement training. Journal of Consulting and Clinical Psychology 65, 703.

Whooley MA, de Jonge P, Vittinghoff E, Otte C, Moos R, Carney RM et al. (2008). Depressive symptoms, health behaviors, and risk of cardiovascular events in patients with coronary heart disease. Journal of the American Medical Association 300, 2379-2388.

\section{Learning objectives}

(1) To illustrate the treatment of a young person with mental health disorders in the context of epilepsy

(2) To demonstrate the effectiveness of a standard treatment protocol for a young person with a complex presentation

(3) To learn about the utility of brief 'simple' interventions to treat mental health disorders in complex populations, such as those with chronic illnesses 